\title{
Development of a Model with a Panel of Biochemical Parameters to Identify Major Depressive Disorder
}

\author{
Soma Gupta ${ }^{1 *}$, Diya Bhaduri², Smarajit Bose ${ }^{3}$, Saswati Nath ${ }^{4}$ and H. N. Das ${ }^{5}$ \\ ${ }^{1}$ Department of Biochemistry, NRS Medical College, Kolkata, India \\ ${ }^{2}$ Department of Statistics, Presidency University, Kolkata, India \\ ${ }^{3}$ Interdisciplinary Statistical Research Unit, Indian Statistical Institute, Kolkata, India \\ ${ }^{4}$ Department of Psychiatry, RG Kar Medical College, Kolkata, India \\ ${ }^{5}$ Department of Biochemistry, RG Kar Medical College (Retired), Kolkata, India
}

\begin{abstract}
Background: There is no available biomarker which can detect cases of Major Depressive Disorder (MDD). So a number of cases remain undetected particularly in those set ups where trained physicians are not available. This case control study was carried out with an aim to find out a panel of parameters in cases of MDD. It was also aimed to find out how successfully people can be classified into MDD and normal groups using this model

Methods: A total number of 150 cases suffering from MDD were included in this study with an equal number of age and gender matched controls. 10 selective biochemical parameters, chosen from review of literature and corroborating pathogenesis of MDD were estimated in blood of all subjects. The parameters, namely, Cortisol, TSH, Prolactin, hsCRP, Ferritin, Cholesterol, Triglyceride, Calcium and Magnesium were estimated by using commercially available kits. Oxidative Stress Index (OSI) was standardized manually. The predictive model was developed using Multiple Logistic Regression. Then the ROC curve analysis of predictive model was done using the software R.

Result: Cholesterol, Triglyceride and Calcium were not considered as they were not found to be significantly altered in MDD. Ferritin was not considered as its level vary according to gender and could not be used as universal parameter. A combined estimation of TSH, Prolactin, hsCRP and Magnesium can identify cases with MDD with $86 \%$ sensitivity and $90 \%$ specificity. Addition of OSI increases the sensitivity to $93 \%$.
\end{abstract}

Conclusion: The model needs validation by further study.

Keywords: Biochemical Parameters, Mdd Identification, Model Development

\section{Introduction}

Globally more than 300 million people suffer from depression, with an increased incidence of $18 \%$, between 2005 and 2015. WHO, with a great concern, gave birth to the slogan, "Depression: let's talk" in April, 2017.

One important reason of this increasing trend of depression is the low diagnosis and treatment rate at different levels of care and in particular at the primary care level. A striking $60 \%$ of depressed patients go undetected at the primary care level and when detected, only $30 \%$ are treated. It has been reported that it is especially difficult to promote and implement the use of new knowledge on depression by clinicians (1). A panel of biochemical parameter may solve this problem even if there is scarcity of properly trained psychiatrists. Another important cause of the increasing incidence of Depression is that, the diagnosis of depression depends entirely upon presence of some symptoms (2) and so far, no known physical test or biochemical parameter is available to diagnose the condition positively. Major Depressive Disorder (MDD) is the commonest form of depression, which was found to affect approximately $3 \%$ of the global population in 2015. Though different theories have been laid down, the exact pathophysiology of depression is yet to be established which also limits the availability of any biochemical parameter (3).

Among the theories regarding pathogenesis of depression, "The Monoamine theory of Depression" suggested the involvement of monoamine neurotransmitters, namely serotonin, dopamine and nor epinephrine. Estimation of these neurotransmitters is difficult and thus their use as a diagnostic tool in depression is limited. Among the other theories, the Neuroendocrine Hypothesis (4) emphasizes on altered endocrine functions. Immune Cytokine mechanism (5) suggests that physical and mental stressors trigger inflammatory processes, activate immune system and release cytokines, which can provoke the moods, 
behavior as well as thoughts characteristics of depression. Nutritional status of vitamins and minerals were also found to play important role, as they are involved in different metabolic regulations. Several studies implicated impairment of energy metabolism pathways in both pathogenesis of depression and effects of antidepressants. Perturbations in lipid metabolism especially cholesterol has been mostly studied in MDD (6). Theory of Oxidative stress in pathogenesis of depression is based on the fact that neurons are vulnerable to free radical attack. Hence insufficient anti-oxidant defense or exposure to excess Reactive Oxygen Species (ROS) can lead to dysfunction of neurons leading to decrease in the volume of hippocampus in patients with major depression and death of neurons (7).

From the available literature, a panel of parameters was chosen which are known to get altered in depression and the cause of alteration can be explained biochemically. Moreover, those parameters, which can be easily estimated in any laboratory, were given priority. The parameters chosen are, cortisol, TSH and prolactin (among theory of neuro endocrine mechanism), hsCRP and ferritin (among immune cytokine mechanism), calcium and magnesium (among theory of alteration of Nutritional status), cholesterol and triglyceride (among theory of metabolic derangement). Oxidative stress index (calculated from total antioxidant capacity and total peroxide concentration), though not commonly estimated in the commercial laboratories, is also included as a parameter as the theory of oxidative stress in depression is stated very strongly. A cheap and manual method is chosen for this purpose, so that it could be easily standardized in any laboratory.

With this background, the present study is undertaken to estimate the concentration of 10 selective parameters in cases of MDD and compare the concentration of same parameters with respect to Non MDD subjects. Then it was aimed to find out whether any model can be prepared out of the significantly altered parameters and if any model can be developed, how successfully people can be classified into MDD and non MDD groups using that model.

\section{Materials and Methods}

This case control study was undertaken in Department of Biochemistry, College of Medicine \& Sagore Dutta Hospital in collaboration with Department of Psychiatry of same Institute. The study period was from July, 2013 to Feb, 2017. The study was approved by Institutional Ethics Committee.

Selection of Study Subjects: All patients who were suspected to suffer from MDD were selected from the
Psychiatry outdoor of College of Medicine \& Sagore Dutta Hospital. These patients were first evaluated by detailed history taking and clinical examination through a structured proforma designed for this study. Then they were screened with WHO Five well being index (8). The raw score was calculated. When raw score was below 13 or if the patient had answered 0 to 1 to any of the 5 items, they were further tested. Patients were diagnosed as having MDD according to the Structured Clinical Interview for DSM-IV, and who scored at least 14 points on Major Depression Inventory (MDI) (9).

The exclusion criteria were significant psychiatric comorbidity, organic mental disorder, mental retardation, bipolar disorder, intake of any psychotropic drugs during and at least 1 week before the study, substance abuse, history of endocrine disorders, pregnancy, postpartum depression and lactation.

Apparently healthy, age and sex matched individuals were assessed using General Health Questionnaire (GHQ 12). A score of less than or equal to 15 were considered as not to suffer from major psychiatric illness (10). Such individuals were selected as control group.

Informed consents were taken from the patients or legal guardians and from the control subjects.

Sample Collection, Separation \& analysis of serum: An amount of $10 \mathrm{ml}$ of fasting blood samples was drawn aseptically from each of the study subjects (both cases and controls), $3 \mathrm{ml}$ was collected in vial containing EDTA as anticoagulant and $7 \mathrm{ml}$ in plain vials, which was allowed to clot. Plasma and serum were separated.

Serum of all patients and controls were investigated for Cortisol, TSH, Prolactin, hsCRP, Ferritin by Immuno Enzymometric assay (EIA) (11), Cholesterol by CHOD PAP method (12), Triglyceride by enzymatic method (13), calcium by using metal complexing dye, cresophthalein (14) and magnesium by calmagite method (15). All these methods were estimated using commercially available kits.

Plasma was used to measure Total Antioxidant Capacity (TAC) by FRAP assay of Benzie \& Strain (slightly modified) and Total Plasma Peroxide (TP) concentrations by FOX2 method (with minor modifications). The ratio percentage of the TP to the total antioxidant capacity gave the OSI, an indicator of the degree of oxidative stress. Reagents were manually prepared for TAC and TP (16).

Statistical Analysis: The concentration of all biochemical parameters were expressed in mean $\pm \mathrm{SD}$ in control group and group containing MDD patients and were compared 
for significance by student's $t$ test. A $p$ value of $<0.05$ was considered to be significant. The statistical analysis was done using MedCalc Statistical Software version 16.4.3 (MedCalc Software bvba, Ostend, Belgium; https://www. medcalc.org; 2016).

The predictive model was developed using Multiple Logistic Regression. Then the ROC curve analysis of predictive model was done. These analyses were done using the software R.

\section{Result}

A total number of 150 cases suffering from MDD were included in this study with an equal number of age and gender matched controls. In each group, 112 subjects were female and 38 were male. Table 1 shows level of biochemical parameters in study groups. Most of the parameters except cholesterol, triglyceride and calcium were found to be significantly altered in cases with MDD. Ferritin and magnesium levels were found to be significantly decreased whereas other parameters like cortisol, prolactin, TSH, hsCRP, OSI were found to be significantly increased.
Table 2 shows gender wise comparison of level of The 7 significantly altered parameters in MDD cases. Most of the parameters were found to have no significant alteration among male or female patients suffering from MDD except ferritin.

Then an attempt was made to develop some model for predicting MDD. First, Multiple Group Logistic Regression method was used to establish predictive relation between combined alteration of biochemical parameters and the diagnosis of MDD. The entry of variables with $\mathrm{P} \geq 0.05$ was refused. Table 3 shows the result of regression analysis. $\mathrm{R}^{2}$ (Coefficient of Determination) is achieved when the values of the indicator variables is regressed for predicted probability of MDD. Then the percent of the observations from this outcome that were correctly classified as such by the multinomial was depicted as Percent correctly classified. The significance of the tests is given by Wald $\mathrm{Z}$ test and Wald $\mathrm{P}$ level $<0.05$ was considered as significant.

Then ROC analysis were performed for each model and table 4 gives the result in terms of Area under curve (AUC $\%$, sensitivity and specificity.

Table 1: Biochemical Parameters in Study Groups

\begin{tabular}{|c|c|c|}
\hline Parameter & Non MDD $(\mathbf{n}=150)$ & MDD (n=150) \\
\hline Cholesterol $(\mathrm{mg} \%)$ & $183.9 \pm 22.9$ & $113.7 \pm 24.2$ \\
\hline Triglyceride $(\mathrm{mg} \%)$ & $108.8 \pm 24.1$ & $10.1 \pm 4.6^{*}$ \\
\hline Cortisol $(\mu \mathrm{g} / \mathrm{dl})$ & $7.7 \pm 2.2$ & $8.5 \pm 6.5^{*}$ \\
\hline TSH $(\mu \mathrm{lU} / \mathrm{ml})$ & $2.9 \pm 1.3$ & $25.4 \pm 24^{*}$ \\
\hline Prolactin $(\mathrm{ng} / \mathrm{ml})$ & $10.2 \pm 4.4$ & $3.3 \pm 2.0^{*}$ \\
\hline hsCRP $(\mathrm{mg} / \mathrm{L})$ & $1.6 \pm 0.8$ & $45.8 \pm 30.9^{*}$ \\
\hline Ferritin $(\mathrm{ng} / \mathrm{ml})$ & $57.1 \pm 27.9$ & $9.3 \pm 0.6$ \\
\hline Calcium $(\mathrm{mg} \%)$ & $9.1 \pm 1.0$ & $1.7 \pm 0.6^{*}$ \\
\hline Magnesium $(\mathrm{mg} \%)$ & $2.1 \pm 0.9$ & $2.1 \pm 0.5^{*}$ \\
\hline OSI & $1.3 \pm 0.3$ & \\
\hline
\end{tabular}

All values are expressed in Mean $\pm S D$. $* p<0.05$, when compared by Student t test

Table 2: Biochemical Parameters in cases of MDD according to gender.

\begin{tabular}{|c|c|c|}
\hline Parameter & Female $(\mathbf{n = 1 1 2})$ & Male $(\mathbf{n}=38)$ \\
\hline Cortisol $(\mu \mathrm{g} / \mathrm{dl})$ & $9.9 \pm 4.5$ & $7.9 \pm 3.9$ \\
\hline TSH $(\mu \mathrm{lU} / \mathrm{ml})$ & $8.8 \pm 7.1$ & $22.8 \pm 14.4$ \\
\hline Prolactin $(\mathrm{ng} / \mathrm{ml})$ & $26.2 \pm 26$ & $2.8 \pm 2$ \\
\hline hsCRP $(\mathrm{mg} / \mathrm{L})$ & $3.5 \pm 1.9$ & $70.7 \pm 47.9^{*}$ \\
\hline Ferritin $(\mathrm{ng} / \mathrm{ml})$ & $37.4 \pm 15.5$ & $1.7 \pm 0.6$ \\
\hline Magnesium $(\mathrm{mg} \%)$ & $1.7 \pm 0.5$ & $2.1 \pm 0.4$ \\
\hline OSI & $2.1 \pm 0.5$ & \\
\hline
\end{tabular}

All values are expressed in Mean $\pm S D .{ }^{*} p<0.05$, when compared by Student t test 
Table 3: Result of Multiple Group Logistic Regression.

\begin{tabular}{|c|c|c|c|c|}
\hline Model & Parameter combination & Model $\mathbf{R}^{2}$ & \% correctly classified & Wald z value \\
\hline 1 & TSH+ hsCRP+ Magnesium & 0.55449 & 88.333 & 1.527 \\
\hline 2 & TSH + cortisol +hsCRP + Magnesium & 0.57897 & 89.667 & $2.742^{*}$ \\
\hline 3 & TSH + Prolactin +hsCRP + Magnesium & 0.59583 & 92.667 & $2.624^{*}$ \\
\hline 4 & 3 hormones + hsCRP+ Magnesium & 0.61713 & 91.667 & $3.543^{*}$ \\
\hline 5 & 3 hormones + hsCRP+ Magnesium + OSI & 0.76857 & 94.667 & $5.345^{*}$ \\
\hline
\end{tabular}

Significant when $* p<0.05$

Table 4: ROC analysis of different models.

\begin{tabular}{|c|c|c|c|c|}
\hline Model & Parameter combination & AUC (\%) & Sensitivity (\%) & Specificity (\%) \\
\hline 1 & TSH+ hsCRP+ Magnesium & 91.65 & 84 & 89 \\
\hline 2 & TSH +cortisol +hsCRP + Magnesium & 91.2 & 83 & 89 \\
\hline 3 & TSH + Prolactin +hsCRP + Magnesium & 92.36 & 86 & 90 \\
\hline 4 & 3 hormones + hsCRP+ Magnesium & 91.38 & 84 & 89 \\
\hline 5 & 3 hormones + hsCRP+ Magnesium + OSI & 97.58 & 93 & 90 \\
\hline
\end{tabular}

\section{Discussion}

The diagnosis of depression, when viewed through the lens of Biochemistry, tends to suggest the underlying cause and pathogenesis of the disease.

Increase in cortisol level in patients with depression is reported by several study groups and it is more or less a consistent finding, which can be explained by the activation of HPA axis. Studies on humans show that the HPA axis is activated in different ways during chronic stress depending on the type of stressor, the person's response to the stressor and other factors (17). In MDD, increase in level of cortisol, caused reduced secretion of intracerebral serotonin. Serotonin is known to inhibit TRH constantly. Due to reduction of serotonin, there is increased TRH concentration in brain tissue. As a consequence, TSH secretion gets stimulated in MDD (18). In addition, CSF levels of somatostatin are reduced in depression. Since TSH secretion is under a constant inhibition of somatostatin (19), the consequence might be a further stimulated TSH secretion.

Another hormone, found to be significantly increased in MDD patients in our study is prolactin. Horrobin (20) proposed that prolactin can be increased in MDD, but George et al did not observe any significant alteration of prolactin level in patients with mild depression (21). TRH is not only known to stimulate TSH but also prolactin. Within the brain, prolactin acts as a neuropeptide to promote physiological responses related to reproduction, stress adaptation, neurogenesis, and neuroprotection. Some of these effects are mediated by the activation of different neuronal signaling systems and ion channels (22).
The increase in hsCRP and ferritin can be explained as follows: first, inflammation may lead to depression. Some studies indicate that proinflammatory cytokines might activate the enzyme indoleamine-2,3-dioxygenase, which leads to decreased production of serotonin. Simultaneously, there is increased production of kynurenic and quinolinic acids, which lead to increased release of glutamate and thereby to decreased production of trophic factors, including brain-derived neurotrophic factor, a factor associated with depression (23). Second, depression may lead to inflammation. Psychological stress activates the HPA axis and sympathetic nervous system, which releases stress hormones. These hormones, together with cytokine release induced by stress, initiate the acute-phase response triggering inflammation. This in turn increases levels of the proinflammatory cytokine interleukin 6 , stimulating the production of acute-phase proteins like CRP (24).

So far Ferritin is concerned; there was significant difference among male and female patients. In a study conducted on 4181 participants, male participants with MDD plus comorbid CHD or hypertension were found to have lower levels of ferritin compared to men without MDD, while in women, results were inconsistent. They concluded that MDD reduced levels of ferritin, transferrin and fibrinogen in CVD in a gender specific way (25). Due to the fact that mean serum ferritin level was lower in depressed students than in healthy ones, some researchers suggest that probably iron plays a role in brain function and the establishment of depressive mood. Iron plays an important role in the oxygenation of brain parenchyma and the synthesis of dopamine, a neurotransmitter of the nervous system (26). 
In MDD defects are observed in different metabolic processes, especially that in lipid metabolism. In this study, we did not find any significant alteration in blood level of cholesterol and triglyceride in MDD cases.

So far bivalent cations are concerned; magnesium level in serum was found to be significantly decreased in MDD cases but no significant change was observed in serum calcium value. The finding of low serum magnesium level is in accordance with the findings of Zieba et al (27) and Kirov et al (28).

Oxidative stress index was found to be significantly high in our study, which demonstrates that oxidative stress is significantly present in patients with MDD. This finding is supported by earlier studies (29).

With these findings, we tried to predict a model by combination of different biochemical parameters, which can be considered as an effective model to predict a case of depression. Cholesterol, triglyceride and Calcium were not considered as they were not found to be significantly altered in MDD. Ferritin was not considered as their levels vary according to gender and could not be used as universal parameter.

Among all other parameters, OSI is treated separately as it is not assayed in conventional laboratories.

Model 1: Analysis of individual biomarkers proves that TSH among endocrinal parameters, hsCRP among immunological parameters and Magnesium among bivalent cations, gave the best result. So Parameters of model 1 was $\mathrm{TSH}+\mathrm{hsCRP}+\mathrm{Mg}$.

Model 2: Cortisol, as is the mainstay of the cause of Depression, so far pathogenesis is concerned; it was included to model 1. Thus, parameters of model 2 were $\mathrm{TSH}+$ cortisol +hsCRP $+\mathrm{Mg}$

Model 3: Prolactin is included to parameters of Model 1. Thus, parameters of model 3 were $\mathrm{TSH}+$ Prolactin $+\mathrm{hsCRP}+\mathrm{Mg}$

Model 4: Here all the 3 hormones have been considered along with hsCRP and Magnesium.

Model 5: Parameters of Model 5 are all parameters of Model 4 along with OSI.

The usefulness of each model is given by Coefficient of Determination $\left(\mathrm{R}^{2}\right)$. All the models except model 1 were found to be significant as given by Wald's $\mathrm{P}$ value at the level 0.05 .

So far percentage of cases correctly classified is concerned, arrangement is as follows: model $5>$ model $3>$ model $4>$ model 2> model 1. ROC curve analysis of the models (Table 4) also shows that model 5 is best with $97.58 \%$ area under curve, 93\% sensitivity and 90\% specificity. Next is model 3 with $92.36 \%$ area under curve, $86 \%$ sensitivity and $90 \%$ specificity followed by model 4 where area under curve is $91.38 \%, 84 \%$ sensitivity and $89 \%$ specificity. Thus if OSI cannot be performed, model 3 seems to be a better alternative. Contradictory to the fact that, though there is little doubts that cortisol plays a causal role in pathogenesis of depression; it is not reflected in the predictive model. The metabolic consequences of excess cortisol may be responsible for this finding.

\section{Conclusion}

Biochemical parameters like Cortisol, TSH, Prolactin, hsCRP, Ferritin, Magnesium and Oxidative stress index (OSI) were found to be significantly altered in cases of MDD. A Gender specific difference was observed in cases of Ferritin only. But to prepare a universal model, six parameters, namely OSI, TSH, hsCRP, Prolactin, Magnesium and cortisol were considered.

These 6 parameters were combined in different ways to develop the predictive model. Moreover it was tried to consider minimum number of tests and achieve optimum level of accuracy. In this study, Model 3, which consisted of TSH, Prolactin, hsCRP and Magnesium was found to be the optimum model with $92.36 \%$ area under curve, $86 \%$ sensitivity and $90 \%$ specificity.

The cut off value for the parameters of Model 3 were as follows: TSH : $4.54 \mathrm{uIU} / \mathrm{ml}$, Prolactin: $15.95 \mathrm{ng} / \mathrm{ml}$, hsCRP: $2.1 \mathrm{mg} / \mathrm{L}$, Magnesium: $1.5 \mathrm{mg} \%$. The values of $\mathrm{TSH}$, Prolactin and hsCRP are supposed to be more than the cut off value whereas the value of magnesium is supposed to be lower than the cut off value in a case with MDD.

The model needs validation by further study.

\section{Acknowledgements}

The authors acknowledge the help and support of Prof. Saibal Mukherjee, Principal NRS Medical College (Former Principal, College of Medicine \& Sagore Dutta Hospital), Prof. Indranil Chakrabarty, Head, Department of Biochemistry and Dr. Somsuvra Chottopadhyay, Assistant Professor, Department of Psychiatry, College of Medicine \& Sagore Dutta Hospital.

\section{Reference}

1. Kohli C, Kishore J, Agarwal P, Singh SV. Prevalence of Unrecognised Depression Among Outpatient Department Attendees of A Rural Hospital in Delhi, India. J Clin Diagn Res 2013; 7(9): 1921. 
2. Strawbridge R, Young AH, Cleare AJ. Biomarkers for depression: recent insights, current challenges and future prospects. Neuropsychiatr Dis Treat 2017; 13: 1245.

3. Lakhan SE, Vieira K, Hamlat E. Biomarkers in psychiatry: drawbacks and potential for misuse. Int Arch Med 2010; 3:1.

4. Carroll BJ, Curtis GC, Mendels J. Neuroendocrine regulation in depression: Discrimination of depressed from nondepressed patients. Arch Gen Psychiatry 2010, 33: 1051.

5. Dowlati Y, Herrmann N, Swardfager W, Liu H, Sham L, Reim EK \& Lanctot KL. A meta-analysis of cytokines in major depression. Biological Psychiatry 2010; 67(5): 446.

6. Carroll D, Ring C, Suter M, Willemsen G. The effects of an oral multivitamin combination with calcium, magnesium, and zinc on psychological well-being in healthy young male volunteers: a double-blind placebo-controlled trial. Psychopharmacology (Berl) 2000; 150:220.

7. Sapolsky RM. The possibility of neuro toxicity in the hippocampus in major depression: a primer on neuron death. Biol Psychiatry 2000; 48:755.

8. Primack BA. The WHO 5 Well Being Index performed the best in screening for depression in primary care. Evid Based Med 2003; 8: 155

9. Bech P, Rasmussen NA, Olsen R, Noerholm V\& Abildgaard W. The sensitivity and specificity of the Major Depression Inventory, using the Present State Examination as the index of diagnostic validity. J Affect Dis 2001; 66:159.

10. Goldberg D. Identifying psychiatric illnesses among general medical patients. British Medical Journal 198; 291: 161.

11. Burtis CA, Ashwood ER, editors, Tietz Fundamentals of Clinical Chemistry, WB Saunders, 5th ed. Philadelphia: $2001 ; 862$.

12. Allain CC, Poon LS, Chan CSG, Richmond W, Fu PC. Enzymatic determination of total serum cholesterol. Clin Chem 1974; 20 (4):470.

13. Bucolo G\& David H. Quantitative determination of serum Triglycerides by the use of enzymes. Clin Chem 1973; 19: 476.

14. Stern J, Lewis WHP. The colorimetric estimation of calcium in serum with $\mathrm{O}$ cresophthalein complexone. Clin Chim Acta 1973; 2: 576.

15. Regulatio Liedtke RJ, Croon G. Automated calmagite measurement of magnesium in serum. Clin Chem 1984; 30:1801.

16. Gupta S, Kunti S, Mondal R, Basu P, Chowdhury K M, Gayen R. Determination of reference limit and evaluation of precision to measure Total Antioxidant Capacity (TAC) by Ferric Reducing Antioxidant Power (FRAP) method. Indian Journal of Basic and Applied Medical Research 2014; 3 (4):308.

17. Dinan TG. Glucocorticoids and the genesis of depressive illness. A psychobiological model. British Journal of Psychiatry 1994; 164:365.

18. Cowen PJ. Cortisol, serotonin and depression: all stressed out? The British Journal of Psychiatry 2002; 180 (2): 99.

19. Gold MS, Pottash AL \& Extein I. Hypothyroidism and depression. Evidence from complete thyroid function evaluation. JAMA 2002; 245(19):1919.

20. Horrobin DF. Prolactin and mental illness. Br J Psychiatry 1974; 124: 456.

21. George A, Boyd AE, Seymour R, Don L. Prolactin levels in Mild Depression. Psychosomatic Medicine 1977; 39 (3): 193.

22. Magdaléna Vaváková, Zde^ka uraIková „Jana Trebatická. Markers of Oxidative Stress and Neuroprogression in Depression Disorder. Oxidative Medicine and Cellular Longevity 2015: 1.

23. Wichers $M$ \& Maes M. The psychoneuroimmunopathophysiology of cytokine induced depression in humans. Int J Neuropsychopharmacol 2002; 5 (4): 375.

24. Raison CL, Capuron L, Miller AH. Cytokines sing the blues: inflammation and the pathogenesis of depression. Trends Immunol 2006; 27(1): 24.

25. Marie Kim Wium-Andersen, David Dynnes Ørsted, Sune Fallgaard Nielsen, MScEE \& Børge Grønne Nordestgaard. Elevated C - reactive protein Levels, Psychological Distress, and Depression in 73, 131 Individuals. JAMA Psychiatry 2013;70(2): 176

26. Beard JD, Connor JR, Jones BC. Iron in the brain. Nutr Rey $1993 ; 51: 157$.

27. Zieba A, Kata R, Dudek D, Schlegel-Zawadzka M, Nowak G. Serum trace elements in animal models and human depression: Part III. Magnesium. Relationship with copper. Hum Psychopharm Clin 2000; 15: 631-5.

28. Kirov GK, Tsachev KN. Magnesium, schizophrenia and manic depressive disease. Neuropsychobiology, 23:79-81.

29. Black CN, Bot M, Scheffer PG, Cuijpers P, Penninx BW. Is depression associated with increased oxidative stress? A systematic review and meta-analysis. Psychoneuroendocrinology 2015; 51: 164. 